\title{
Indicadores de sustentabilidad: huella ecológica y capacidad de carga local. El caso de General Pueyrredón ${ }^{1}$
}

\author{
María Cecilia Gareis ${ }^{2}$ \\ Universidad Nacional de Chilecito/Consejo Nacional de Investigaciones Científi- \\ cas y Técnicas/ Universidad Nacional de Mar del Plata \\ @ [ gareiscecilia@gmail.com ]
}

Cita sugerida: Gareis, M. C. (2021). Indicadores de sustentabilidad: huella ecológica y capacidad de carga local. El caso de General Pueyrredón. Revista Huellas, Volumen 25, № 2, Instituto de Geografía, EdUNLPam: Santa Rosa. Recuperado a partir de: http://cerac.unlpam.edu.ar/index.php/huellas

DOI: http://dx.doi.org/10.19137/huellas-2021-2523

\begin{abstract}
Resumen
Entre los indicadores biofísicos que relacionan la disponibilidad de recursos de un territorio con el consumo que de ellos realiza una población, se encuentra la Huella Ecológica (HE) y la Capacidad de Carga Local (CCL). En forma conjunta dan cuenta del grado de sustentabilidad de un territorio. El objetivo del estudio es analizar la situación de sustentabilidad del partido de General Pueyrredón y su ciudad cabecera (Mar del Plata) a partir de la lectura conjunta de dos indicadores: HE y CCL. La metodología desarrollada considera los lineamientos propuestos por Wackernagel y Rees (1996), ajustada a las características de la zona de interés, luego se estima la CCL y se analizan las relaciones entre ambos indicadores. Los resultados muestran una demanda que excede la CCL con la que cuenta el partido de General Pueyrredón, particularidad que expone una situación de déficit ecológico al ser la HE mayor a la CCL, debiéndose cubrir parte de la demanda local con capacidad de carga apropiada (CCA) de otros ecosistemas (importación de biocapacidad).
\end{abstract}

Palabras clave: sustentabilidad, economía ecológica, indicadores biofísicos, déficit ecológico.

\section{Sustainability indicators: ecological footprint and local bearing capacity. The General Pueyrredon Case}

\footnotetext{
Abstract

Among the biophysical indicators that link the availability of resources in a territory with the consumption of those resources by the population are the
} 
Ecological Footprint (EF) and the Local Bearing Capacity (LBC), which together suggest the degree of sustainability of a territory. The aim of this study is to analyze the sustainability situation of the General Pueyrredon district and its capital city (Mar del Plata). The analysis comprises two indicators: EF and LBC. The methodology applied takes into account Wackernagel and Rees' studies (1996), which were adapted to the characteristics of the area, to estimate the LBS afterwards. A final step consisted in analyzing the relationships between both indicators. The results showed that the demand of resources exceeds the LBC of the General Pueyrredon district because the EF is greater than the LBC. Therefore, part of the local demand is imported (LBC) from other ecosystems (import of biocapacity).

Keywords: sustainability; ecological economy; biophysical indicators; ecological deficit

\section{Indicadores de sustentabilidade: pegada ecológica e capacidade de carga local. 0 caso de General Pueyrredón}

\section{Resumo}

Entre os indicadores biofísicos que relacionam a disponibilidade de recursos de um território com o consumo que deles realiza a população se encontra a Pegada Ecológica (PE) e a Capacidade de Carga Local (CCL). De maneira conjunta advertem o grau de sustentabilidade de um território. 0 objetivo do estudo é analisar a situação de sustentabilidade de General Pueyrredón e sua principal cidade (Mar del Plata) a partir da leitura de dois indicadores: PE e CCL. A metodologia desenvolvida considera os alinhamentos propostos por Wackernagel e Rees (1996) ajustada às características da zona de interesse, logo se estima a CCL e se analisam as relações entre ambos indicadores. Os resultados mostram uma demanda que excede a CCL com a que conta o partido de General Pueyrredón, particularidade que expõe uma situação de déficit ecológico ao ser a PE maior à CCL, devendo-se cobrir parte da demanda local com a capacidade de carga apropriada (CCA) de outros ecossistemas (importação de biocapacidade).

Palavras-chave: Sustentabilidade; economia ecológica; indicadores biofísicos; déficit ecológico 


\section{Introducción}

El aporte se encuadra en los postulados teóricos de la Economía Ecológica, presentados y analizados en trabajos previos (Gareis, 2017, Gareis y Ferraro, 2018, Gareis y Ferraro, 2019). La metodología base desarrollada por Wackernagel y Rees (1996) fue ajustada para el caso en estudio para la estimación de la HE (Gareis, 2017, Gareis y Ferraro, 2018, Gareis y Ferraro, 2019). A partir de allí se establecen las categorías a los fines de comparar las subhuellas que componen a la HE con las subcapacidades que conforman la CCL del partido de General Pueyrredón (Figura $N^{\circ} 1$ ). Esto permite contrastar cuánto de lo que consume la población local (a nivel de ciudad y partido), que se refleja en los valores parciales que toma cada subhuella, puede abastecerse por medio de las hectáreas productivas con las que cuenta el partido atendiendo a la disponibilidad de cada subcapacidad de la CCL. El objetivo del estudio es analizar la situación de sustentabilidad del partido de General Pueyrredon y su ciudad cabecera (Mar del Plata) a través de la lectura conjunta de dos indicadores biofísicos: Huella Ecológica (HE) y Capacidad de Carga Local (CCL).

En este estudio se pretende avanzar en el análisis de la relación HECCL que se efectúa en la etapa final de una investigación doctoral, que permite comparar dos indicadores biofísicos y efectuar lecturas generales en términos de sustentabilidad (en el sentido fuerte de la palabra).

Figura $\mathbf{N}^{\circ}$ 1. Localización del partido de General Pueyrredón y ciudad de Mar del Plata

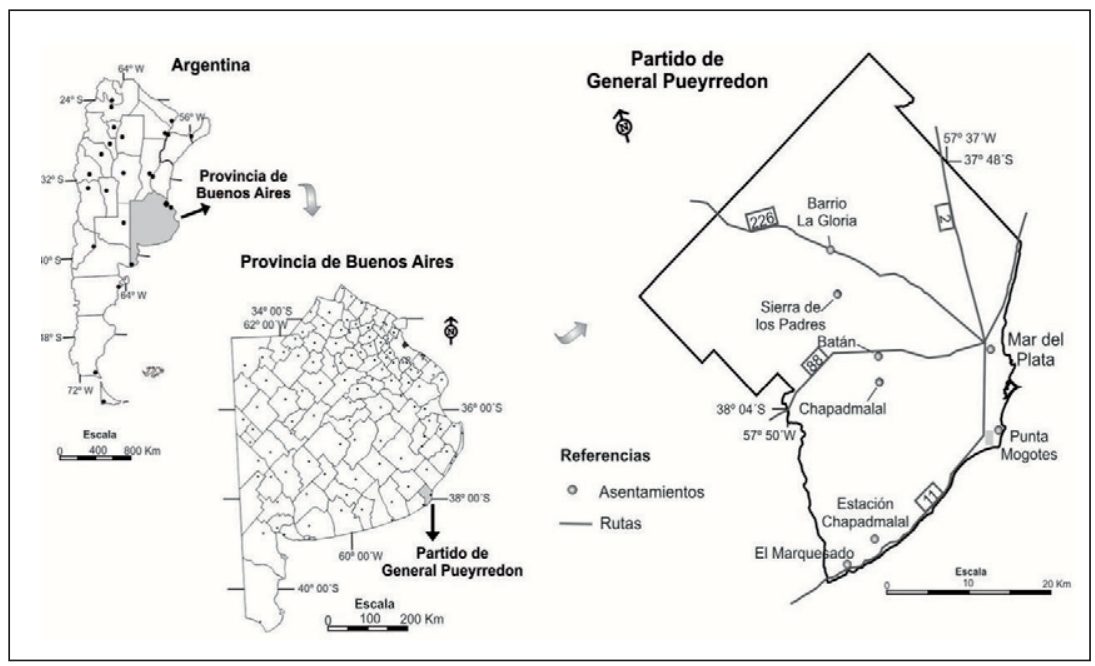

Fuente: Ferraro et al. (2013). 


\section{Materiales y métodos}

Si bien se empleó como base la propuesta metodológica original (Wackernagel y Rees, 1996) para la estimación de la HE, se realizaron una serie de ajustes generales que se mencionan en trabajos antecedentes (Gareis, 2017, Gareis y Ferraro, 2018). Las modificaciones se realizaron al indicador principalmente por cuatro razones fundamentales y que condicionaron su cálculo: disponibilidad de información, características en la que se presentan los datos, particularidades del partido de General Pueyrredón y objetivos del estudio.

\section{Estimación de la CCL del partido de General Pueyrredón}

Luego de calcular la HE fue necesario estimar la CCL a los fines de evaluar la situación del partido y ciudad cabecera en términos de sustentabilidad. Así, lo que se pretende es determinar en qué proporción cada una de las subhuellas que conforman la HE es "cubierta" por la capacidad de carga local que se vincula a cada subhuella.

Para ello, se trabajó con información agrícola del Departamento de Información Estratégica de la Municipalidad de General Pueyrredón, correspondiente a la campaña 2010/2011 y se analizó la superficie destinada a la agricultura extensiva e intensiva y la ganadería, ya que son la base de todos los alimentos. Se descontaron los alimentos comercializados al exterior del partido, en carácter de "capacidad de carga apropiada o robada", únicamente en el caso de la horticultura porque se contaba con la información. En lo que respecta a la ganadería se trabajó además con información obtenida del MAGyP (2013, p. 45, p. 99, p. 119, p. 165, p. 213).

La CCL se estimó de cara a cada una de las subhuellas analizadas, esto es, se calculó para cada una de ellas la subcapacidad de carga local (SCCL).

\section{SCCL vinculada a la absorción de $\mathrm{CO}_{2}$}

Para el caso de la SCCL asociada a la absorción de $\mathrm{CO}_{2}$, se consideraron los distintos usos de suelo, se identificó y calculó la superficie de cada uno, se empleó como base la clasificación realizada por Zulaica et al. (2011) para el año 2009 y se asumieron como válidos esos cálculos para el año 2010. Las categorías utilizadas por los autores fueron: Agricultura hortícola al aire libre, agricultura hortícola bajo cubierta, agricultura extensiva, urbana, industrial, minera, ganadera y áreas con agua. En lo que respecta al cálculo de la superficie, se consideraron los valores de las ac- 
tividades urbanas, de disposición de residuos (estas áreas se unificaron), industriales, mineras, ganaderas y áreas de agua.

También se apeló a información obtenida de la página oficial del partido de General Pueyrredón referente a la superficie destinada a la horticultura al aire libre y bajo cubierta (campaña 2010/2011) y a datos de la Dirección de Información Agrícola y Forestal (2011) correspondientes a la superficie destinada a cultivos extensivos (campaña 2010/2011). Asimismo, de la diferencia entre la superficie correspondiente al conjunto de las actividades identificadas y la superficie del partido, se agregó la categoría "otros" para incluir las superficies que no tienen un uso definido. De la sumatoria se obtuvo el área total del partido.

Para la estimación de la capacidad de absorción de $\mathrm{CO}_{2}$ del partido de General Pueyrredón, se consideraron los lineamientos propuestos por Rodríguez Mellado (2008) y se agruparon en actividades los distintos usos de suelo y los valores de absorción correspondientes a cada uno (se promediaron), para poder estimarla según actividad, considerando únicamente aquellas que se adecuaban a las particularidades locales. A partir de allí fue posible determinar la absorción de $\mathrm{CO}_{2}$ considerando la particularidad de cada tipo de uso de suelo, como así también el valor medio de absorción para el año 2010. Se obtuvo la subhuella per cápita a partir de vincular lo emitido per cápita con lo absorbido por una hectárea promedio del partido de General Pueyrredón.

\section{SCCL vinculada al ambiente construido}

Para el caso de la SCCL asociada al ambiente construido, se consideró como tal a la superficie destinada por medio del Código de Ordenamiento Territorial del partido de General Pueyrredón (COT) a uso urbano. Para ello se estimó el área prevista para ese uso coincidente con la superficie máxima a destinarse a ambiente construido.

\section{SCCL vinculada a alimentos}

Para determinar la SCCL vinculada a los alimentos se realizó el cálculo para aquellos de origen terrestre y marino. En el primer caso se recopiló información antecedente y se analizó la superficie destinada a la agricultura extensiva, intensiva (horticultura) y a la ganadería. Luego se descontaron los alimentos comercializados por fuera de los límites del partido en carácter de "capacidad de carga apropiada o robada" (caso de alimentos hortícolas y superficie destinada a la producción de colza y avena). Para el segundo caso se consideró, en primera medida, el área correspondiente a la plataforma marina que correspondería al partido, si se parte de considerar 
que hay una distancia aproximada de 187 kilómetros desde el puerto de Mar del Plata hasta los 200 metros bajo el nivel del mar. Dicha distancia constituye el límite establecido de la plataforma marina que correspondería a Argentina y, en este caso, al partido de General Pueyrredón por tener costa. De esta manera, se determinó el área que luego se vinculó al valor de productividad promedio establecido en 33,1 kg de pez por hectárea por Wackernagel y Rees (1996, p. 65). Luego se sumaron los valores parciales y se logró estimar la SCCL per cápita que se ajustó a nivel de población del partido (con y sin turistas) y ciudad de Mar del Plata.

\section{SCCL vinculada a papel y madera}

En el caso del papel y la madera, no fue posible determinar la SCCL que pueda luego vincularse a la SHMP por no producirse dichos bienes dentro del partido, razón por la cual se le atribuyó un valor de Oha a este ítem.

\section{SCCL vinculada a la absorción de residuos}

En cuanto a la SCCL vinculada a la absorción de residuos, se calculó a partir de considerar el potencial de acogida que presenta el Centro de Disposición Final de Residuos Sólidos Urbanos (CDFRSU). El relleno sanitario consta de 2 módulos y cada uno de ellos agrupa un conjunto de sectores. El módulo 1 ocupa una superficie de 21,5 ha y una capacidad de $2.524 .560 \mathrm{~m}^{3}$ y el módulo 2 de 18,4 ha y $2.391 .720 \mathrm{~m}^{3}$ de capacidad. Por lo tanto, la capacidad total de recepción de residuos es de 4.916.280m³ (Dirección General de Gestión Ambiental, 2012, p. 61). Si se considera que como mínimo se debe alcanzar una densidad de $0,9 \mathrm{t} / \mathrm{m}^{3}$ (Dirección General de Gestión Ambiental, 2012, p. 69; Dirección General de Gestión Ambiental, 2013, p. 68), es posible determinar la capacidad de recepción total que tendría el relleno. La superficie directamente destinada a la recepción de residuos es de 39,9ha, valor que surge de sumar las hectáreas correspondientes a cada módulo. A partir de allí se estimó la SCCL asociada a los residuos a partir de vincular la capacidad total del relleno con las hectáreas destinadas a tal. Luego se procedió a determinar la CCL per cápita, a nivel de población del partido de General Pueyrredon (incluida la población de turistas) y ciudad de Mar del Plata.

\section{CCL total}

Finalmente, la CCL total del partido de General Pueyrredón se estimó a partir de sumar las SCCL parciales. 


\section{Relación entre la Huella Ecológica y la Capacidad de Carga Local}

Por último, se vincularon los resultados del cálculo de la $\mathrm{HE}$ a nivel de población del partido de General Pueyrredón (con y sin turistas) y ciudad de Mar del Plata con las estimaciones efectuadas sobre la CCL (Figura $\mathrm{N}^{\circ}$ 2), a los fines de realizar una lectura integral y analizar la situación del partido en términos de sustentabilidad, desde los postulados teóricos de la Economía Ecológica.

Figura $\mathbf{N}^{\circ}$ 2. Esquematización de la relación metodológica entre HE y CCL
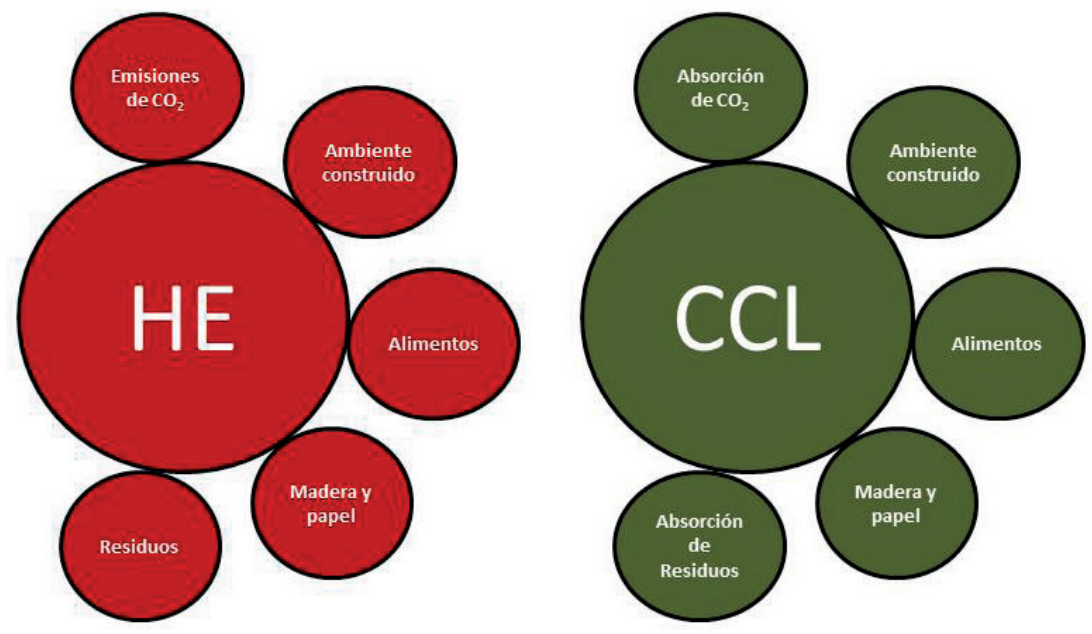

Fuente: Elaboración propia.

\section{Huella Ecológica del partido de \\ General Pueyrredón y ciudad de Mar del Plata}

Existen trabajos antecedentes que avanzan en la explicación teórica y metodológica de la estimación de la HE para General Pueyrredón (Gareis, 2017), como así también en los resultados a nivel de subhuellas que la componen (Gareis y Ferraro, 2013, Gareis y Ferraro, 2014, Gareis y Ferraro, 2015, Gareis et al., 2016, Gareis, 2017). A los fines de establecer comparaciones claras entre la $\mathrm{HE}$ y la $\mathrm{CCL}$ es que se presentan sintéticamente los datos agregados a nivel de subhuellas en la Figura $\mathrm{N}^{\circ} 3$ y el Cuadro $N^{\circ} 1$ a nivel de partido de General Pueyrredón con y sin turistas y a escala de ciudad de Mar del Plata.

La población de General Pueyrredón, estimada por el INDEC (2010) en 618.989 habitantes para el año 2010, demandó en conjunto 1.153.133,27 
hectáreas productivas para cubrir sus necesidades energéticas y materiales aquí contempladas y absorber, a su vez, los residuos generados como resultado del consumo efectuado. Ese valor asciende a 1.302.652,06 hectáreas de considerar a la población de turistas, lo que representa un incremento del $13 \%$, mientras que para el caso de Mar del Plata las hectáreas productivas son menores, pero apenas en un 0,52\%. Esta diferencia se debe a que el 95\% de la población del partido se ubica en zonas urbanas.

Cuadro $N^{\circ} 1$. Resumen de la HE según SH a nivel per cápita, partido de General Pueyrredón (con y sin turistas) y ciudad de Mar del Plata

\begin{tabular}{|c|c|c|c|c|c|}
\hline SH & $\begin{array}{l}\text { Per cápita } \\
\text { (ha/cap) }\end{array}$ & $\begin{array}{l}\text { Población del } \\
\text { partido sin turistas } \\
\text { (ha/pobl) }\end{array}$ & $\begin{array}{c}\text { Población del } \\
\text { partido con turistas } \\
\text { (ha/pobl) }\end{array}$ & $\begin{array}{l}\text { Población de Mar } \\
\text { del Plata (ha/pobl) }\end{array}$ & SH $\%$ \\
\hline $\begin{array}{l}\text { Emisiones } \\
\text { de } \mathrm{CO}_{2}\end{array}$ & 0,73 & $454.169,39$ & $513.058,38$ & $451.778,91$ & 39,4 \\
\hline $\begin{array}{l}\text { Ambiente } \\
\text { construido }\end{array}$ & 0,031 & $19.549,19$ & 22.084 & $19.446,3$ & 1,7 \\
\hline Alimentos & 1,04 & $644.535,97$ & $728.108,47$ & $641.143,5$ & 55,9 \\
\hline $\begin{array}{l}\text { Madera y } \\
\text { papel }\end{array}$ & 0,056 & $34.876,37$ & $39.398,55$ & $34.692,80$ & 3 \\
\hline Residuos & 0,0000038 & 2,35 & 2,66 & 2,34 & 0,0002 \\
\hline HE (ha) & 1,86 & $1.153 .133,27$ & $1.302 .652,06$ & $1.147 .063,85$ & 100 \\
\hline
\end{tabular}

Fuente: Gareis (2017), Gareis y Ferraro (2019).

En síntesis, la demanda per cápita se ubica en 1,86 hectáreas. Esto significa que el habitante promedio del partido de General Pueyrredón requirió de 1,86 hectáreas productivas para, por un lado, abastecerse de alimentos, madera y papel y habitar y para que, por otro lado, se absorban las emisiones de $\mathrm{CO}_{2}$ y residuos producto del consumo energético y de materia.

Si se considera que el partido cuenta con una superficie total de aproximadamente 146.000 hectáreas, los resultados aquí presentados dan cuenta de una superficie apropiada que multiplica en 7,90 veces el área administrativa del partido, que asciende a 8,92 si se incluye la demanda ejercida por la población turística y desciende a 7,86 si sólo se considera la ciudad de Mar del Plata.

Sin embargo, las cifras mencionadas $(7,90,8,92$ y 7,86$)$ descienden sensiblemente al considerar en el valor de la superficie total la correspondiente al mar (785.400 hectáreas estimativamente), para dar un valor final de 931.400 hectáreas. Por lo tanto, el valor de HE para la población 
del partido, de considerar la población turística, es 0,7 veces mayor a la superficie productiva total destinada a satisfacer las necesidades de la población en estudio.

Figura $\mathbf{N}^{\circ}$ 3. Huella ecológica del partido de General Pueyrredon

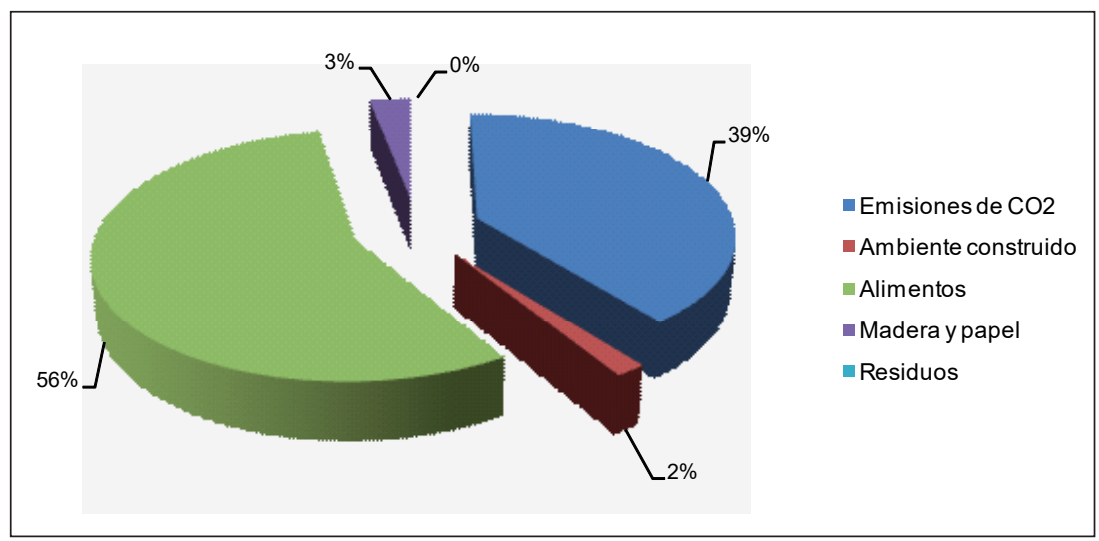

Fuente: Gareis (2017), Gareis y Ferraro (2019).

\section{Estimación integral de la CCL total para el partido de General Pueyrredón y análisis particular de su ciudad cabecera}

De la sumatoria de las subcapacidades que conforman la CCL se obtuvo el valor total para el partido de General Pueyrredón. De esta manera se observa (Figura $N^{\circ} 4$ ) que la participación mayor la ejerce el área productiva destinada a los fines de producción de alimentos que conforma el $84 \%$ del valor de la CCL total. Mientras que no tiene ninguna incidencia en la CCL la producción de madera y papel por no emplearse áreas a estos fines dentro del partido, siendo muy baja la cantidad de hectáreas destinadas a la absorción de residuos.

Es importante mencionar que la CCL asociada a la producción de alimentos está fuertemente influenciada por la productividad marina, el valor es considerablemente más bajo de sólo contemplar los alimentos de origen terrestre $(0,17 \mathrm{CCL} / \mathrm{cap})$, en términos comparativos $(1,12 \mathrm{CCL} / \mathrm{cap}$ para el caso de los alimentos de origen marino).

Como se observa en el Cuadro No 2 , la CCL del partido de General Pueyrredón es de 1.078.579,46 hectáreas productivas totales de considerar a la población turística, que de descontarse muestra una CCL disponible para la población local del partido de 954.779,62 hectáreas, por lo tanto, la población turística se apropia de 123.799,84 hectáreas productivas de la CCL total. 
Cuadro $\mathbf{N}^{\circ}$ 2. Resumen de la CCL según SH a nivel per cápita, partido de General Pueyrredón (con y sin turistas) y ciudad de Mar del Plata

\begin{tabular}{|c|c|c|c|c|c|}
\hline CCL & $\begin{array}{c}\text { Per cápita } \\
\text { (CCL/cap) }\end{array}$ & $\begin{array}{c}\text { Población del } \\
\text { partido sin turistas } \\
\text { (CCL/pobl) }\end{array}$ & $\begin{array}{c}\text { Población del } \\
\text { partido con turistas } \\
\text { (CCL/pobl) }\end{array}$ & $\begin{array}{c}\text { Población de } \\
\text { Mar del Plata } \\
\text { (CCL/pobl) }\end{array}$ & CCL \% \\
\hline $\begin{array}{c}\text { Absorción } \\
\text { de CO }\end{array}$ & 0,22 & $129.242,08$ & 146.000 & $128.561,82$ & 13,3 \\
\hline $\begin{array}{c}\text { Ambiente } \\
\text { construido }\end{array}$ & 0,035 & 21.724 & 24.541 & 21.610 & 0,02 \\
\hline Alimentos & 1,29 & $803.778,22$ & $907.998,56$ & $799.547,6$ & 84,42 \\
\hline $\begin{array}{c}\text { Madera y } \\
\text { papel }\end{array}$ & 0 & 0 & 0 & 0 & 0 \\
\hline Residuos & 0,000057 & 35,32 & 39,9 & 35,13 & 0,004 \\
\hline CCL (ha) & 1,54 & $954.779,62$ & $1.078 .579,46$ & $949.754,55$ & 100 \\
\hline
\end{tabular}

Fuente: Gareis (2017).

En síntesis, el habitante del partido de General Pueyrredón dispone de 1,54 hectáreas productivas para satisfacer sus necesidades anuales de materia y energía, en este caso de alimentos, energía, para la absorción de residuos y emisiones de $\mathrm{CO}_{2}$ y habitar.

Figura № 4. CCL del partido de General Pueyrredón

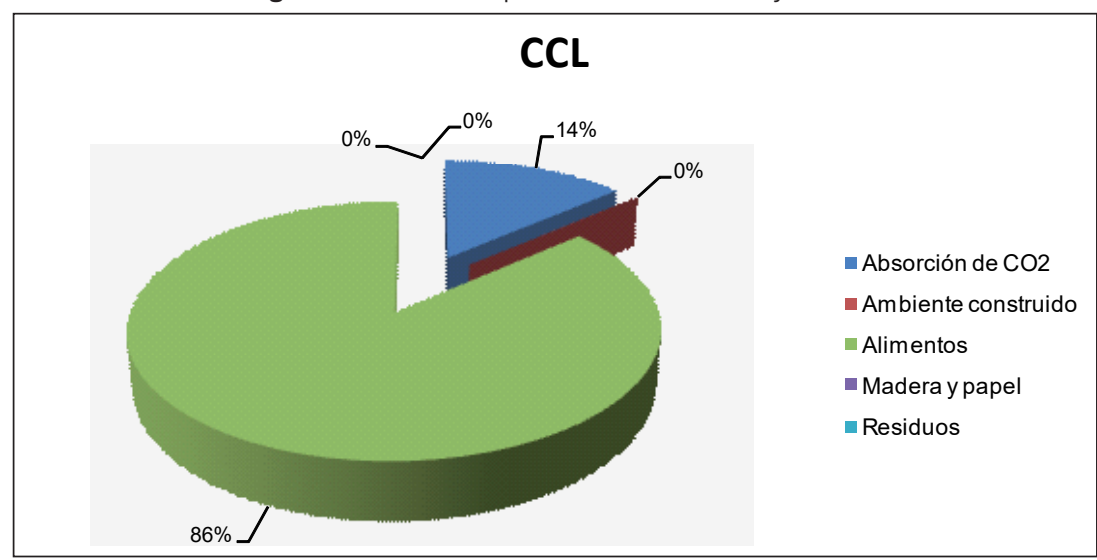

Fuente: Gareis (2017).

De considerarse que el partido presenta una superficie estimada en 146.000 hectáreas aproximadamente, los resultados correspondientes a la CCL superan en 7,4 veces el área administrativa de éste. Sin embargo, 
debe considerarse aquí la CCL asociada a la producción de alimentos de origen marino estimada en 785.400 hectáreas aproximadamente, lo que arroja un valor final de 931.400 hectáreas productivas entre lo terrestre y lo marino. Así, la CCL total excede en 147.179 hectáreas la superficie terrestre y marina que presenta el partido.

\section{Comparación de los resultados a niveles: per cápita, partido (con y sin turistas) y ciudad cabecera}

Una lectura general a nivel de subhuella y su relación con la CCL, a niveles per cápita, muestra (en la Figura $\mathrm{N}^{\circ} 5$ ) que para los casos del ambiente construido, alimentos y residuos, los valores que tomaron las subhuellas son menores comparativamente a las SCCL para esos mismos ítems, lo que permite decir que la demanda per cápita que se ejerce en el partido no supera la disponibilidad local. Por el contrario, para los casos de absorción de $\mathrm{CO}_{2}$ y madera y papel se observa una relación negativa, esto es, los valores que toman las subhuellas mencionadas exceden los de las SCCL.

Figura Nº 5. CCL y HE per cápita del partido de General Pueyrredón a nivel de subhuellas, subcapacidades y valores totales

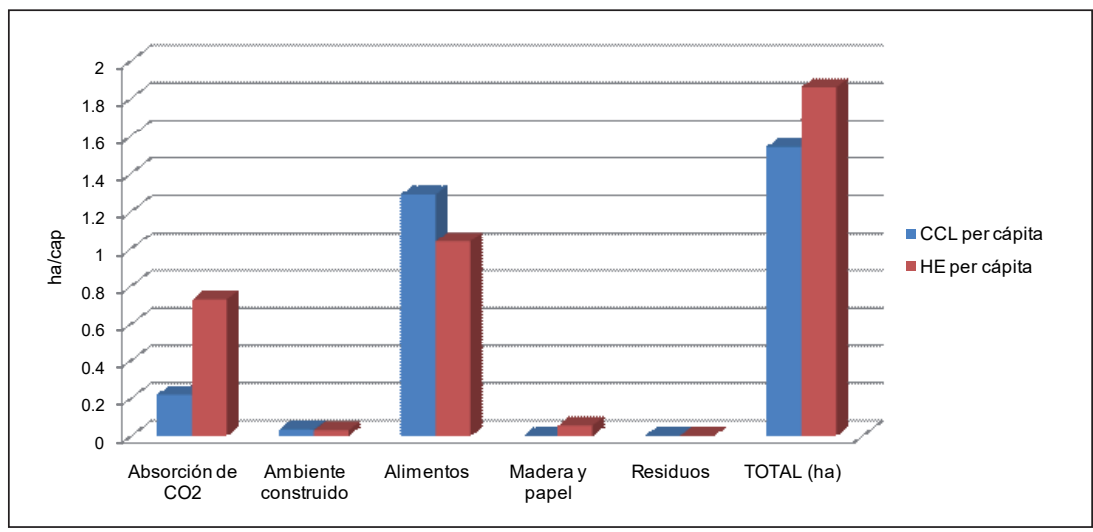

Fuente: Gareis (2017).

Lo antes mencionado se observa con mayor detalle en el Cuadro $N^{\circ}$ 3 , en donde se vincula la CCL con la HE y se muestran las diferencias en hectáreas. 
Cuadro № 3. Relación entre CCL y HE del partido de General Pueyrredón a nivel per cápita.

\begin{tabular}{|l|c|c|c|c|}
\hline & $\begin{array}{c}\mathrm{CCL} \\
\text { per cápita }\end{array}$ & $\begin{array}{c}\mathrm{HE} \\
\text { per cápita }\end{array}$ & $\begin{array}{c}\mathrm{CCL}-\mathrm{HE} \\
\text { (ha/cap) }\end{array}$ & $\begin{array}{c}\mathrm{HE} / \mathrm{CCL} \\
\text { (ha/cap) }\end{array}$ \\
\hline Absorción de $\mathrm{CO}_{2}$ & 0,22 & 0,73 & $-0,51$ & 3,32 \\
\hline Ambiente construido & 0,035 & 0,031 & 0,004 & 0,89 \\
\hline Alimentos & 1,29 & 1,04 & 0,25 & 0,81 \\
\hline Madera y papel & 0 & 0,056 & $-0,056$ & \\
\hline Residuos & 0,000057 & 0,0000038 & 0,0000532 & 0,07 \\
\hline TOTAL (ha) & 1,54 & 1,86 & $-0,32$ & 1,21 \\
\hline
\end{tabular}

Fuente: Gareis (2017).

De la relación entre los valores obtenidos por las subhuellas se observa que la HE resulta ser superior a la $\mathrm{CCL}$, lo que da cuenta de una situación de (in)sustentabilidad, debido a que la población requiere de una cantidad mayor de hectáreas productivas de las que éste dispone para abastecer la demanda. Razón por la cual es necesaria la importación de bienes y servicios de ecosistemas que se ubican por fuera de los límites del partido. Por ello, el partido en estudio "importa" CCL de otros sitios.

Cuadro $N^{\circ}$ 4. Relación entre CCL y HE del partido de General Pueyrredón a nivel de población local sin considerar a los turistas

\begin{tabular}{|l|c|c|c|}
\hline & $\begin{array}{c}\text { CCL PGP - turistas } \\
\text { (ha/pobl) }\end{array}$ & $\begin{array}{c}\text { HE PGP - turistas } \\
\text { (ha/pobl) }\end{array}$ & $\begin{array}{c}\text { CCL - HE } \\
\text { (ha/pobl) }\end{array}$ \\
\hline Absorción de $\mathrm{CO}_{2}$ & $129.242,08$ & $454.169,39$ & $-324.927,31$ \\
\hline Ambiente construido & 21.724 & $19.549,19$ & $2.174,81$ \\
\hline Alimentos & $803.778,22$ & $644.535,97$ & $159.242,25$ \\
\hline Madera y papel & 0 & $3.487,37$ & $-34.876,37$ \\
\hline Residuos & 35,32 & 2,35 & 32,97 \\
\hline TOTAL (ha) & 954.779 .62 & $1.153 .133,27$ & $-198.353,65$ \\
\hline
\end{tabular}

Fuente: Gareis (2017).

La situación mencionada para los valores per cápita (Cuadro $N^{\circ} 4$ ) se observan también cuando se compara la HE y la CCL a nivel de población, al descontar la incidencia de los turistas. En este sentido, se evidencia un déficit ecológico de 198.353,65 hectáreas. 
Cuadro $N^{\circ}$ 5. Relación entre CCL y HE del partido de General Pueyrredón a nivel de población local considerando a los turistas.

\begin{tabular}{|l|c|c|c|}
\hline & $\begin{array}{c}\text { CCL PGP + turistas } \\
\text { (ha/pobl) }\end{array}$ & $\begin{array}{c}\text { HE PGP + turistas } \\
\text { (ha/pobl) }\end{array}$ & $\begin{array}{c}\text { CCL - HE } \\
\text { (ha/pobl) }\end{array}$ \\
\hline Absorción de $\mathrm{CO}_{2}$ & 146.000 & $513.058,38$ & $-367.058,38$ \\
\hline Ambiente construido & 24.541 & 22.084 & 2.457 \\
\hline Alimentos & $907.998,56$ & $728.108,47$ & $179.890,09$ \\
\hline Madera y papel & 0 & $39.398,55$ & $-39.398,55$ \\
\hline Residuos & 39,9 & 2,66 & 37,24 \\
\hline TOTAL (ha) & $1.078 .579,46$ & $1.302 .652,06$ & $-224.072,6$ \\
\hline
\end{tabular}

Fuente: Gareis (2017).

El déficit ecológico se incrementa de considerar la población asociada al turismo a 224.072,6 hectáreas (Cuadro $N^{\circ}$ 5), mientras que se reduce a 197.309,3 hectáreas si sólo se considera a la ciudad de Mar del Plata (Cuadro $N^{\circ} 6$ ).

Cuadro № 6. Relación entre CCL y HE del partido de General Pueyrredón a nivel de ciudad de Mar del Plata.

\begin{tabular}{|l|c|c|c|}
\hline & $\begin{array}{c}\text { CCL Mar del Plata } \\
\text { (ha/pobl) }\end{array}$ & $\begin{array}{c}\text { HE Mar del Plata } \\
\text { (ha/pobl) }\end{array}$ & $\begin{array}{c}\text { CCL - HE } \\
\text { (ha/pobl) }\end{array}$ \\
\hline Absorción de $\mathrm{CO}_{2}$ & $128.561,82$ & $451.778,91$ & $-323.217,09$ \\
\hline Ambiente construido & 21.610 & $19.446,3$ & $2.163,7$ \\
\hline Alimentos & $799.547,6$ & $641.143,5$ & $158.404,1$ \\
\hline Madera y papel & 0 & $34.692,8$ & $-34.692,8$ \\
\hline Residuos & 35,13 & 2,34 & 32,79 \\
\hline TOTAL (ha) & $949.754,55$ & $1.147 .063,85$ & $-197.309,3$ \\
\hline
\end{tabular}

Fuente: Gareis (2017).

\section{Conclusiones}

Desde la perspectiva de la Economía Ecológica (EE), una sociedad es sustentable en la medida en que satisface el consumo per cápita de su población sin menoscabar el capital esencial de stock de una generación a la siguiente. No obstante, y tal como lo mencionan Rees y Wackernagel (1996), ninguna ciudad o región urbana puede alcanzar la sustentabilidad por sí sola sin efectuar un uso sustentable del hinterland mundial o global.

El presente trabajo da cuenta de la agotabilidad de los recursos y de un uso mayor a la capacidad de carga local que presenta General Pueyrredón lo que muestra una situación de déficit ecológico al ser la HE mayor a la 
$\mathrm{CCL}$, debiéndose cubrir la demanda local con capacidad de carga apropiada o robada a otros ecosistemas ubicados por fuera del partido, lo que se denomina también importación de biocapacidad.

Wackernagel y Rees plantean como deseable la idea de incrementar la independencia regional a los flujos externos que presentan las regiones urbanas o países, a los fines de crear una especie de protección ante la creciente demanda internacional de bienes. A la vez que plantean como necesaria la elaboración de políticas urbanas que tiendan a reducir la interrupción humana sobre los ecosistemas y el consumo de energía y materiales que realizan las ciudades.

En este sentido la HE permite visualizar algunos aspectos, pero no da la información precisa y necesaria a los fines de justificar políticas que puedan realizarse en este sentido. Asimismo, en las actuales tendencias de globalización, resulta cada vez más alejada la idea de concebir los espacios "desconectados" de la influencia externa, tanto de flujos de materiales como de energía. No obstante, es necesario trabajar en políticas de desarrollo local que tengan en cuenta estas consideraciones tendientes a elaborar instrumentos de gestión que se ajusten a la particularidad local.

Aplicar políticas en pos de trabajar en algunos de los aspectos identificados como relevantes en HE debe concebirse desde la perspectiva de sistema en pos de crear políticas que actúen a modo de "multiplicador de sustentabilidad urbana" (Rees, 1996).

En síntesis, estimar la HE y la CCL permiten evaluar la situación de sustentabilidad en la que se presenta el partido de General Pueyrredón. Los resultados permiten afirmar que la apropiación de ecosistemas por parte de la población del partido supera la capacidad de sustentación del mismo.

\section{Referencias bibliográficas}

Dirección General de Gestión Ambiental. (2012). Informe anual ambiental 20112012. Partido de Gral. Pueyrredon, Mar del Plata, Buenos Aires-Argentina. Recuperado de http://www.mardelplata.gob.ar/documentos/gestionambiental/informe_anual_ambiental_final\%202012.pdf

Dirección General de Gestión Ambiental. (2013). Informe anual ambiental 2013. Municipalidad de General Pueyrredon. Recuperado de http://www.mardelplata.gob. ar/documentos/enosur/informe\%20ambiental\%20anual\%202013.pdf
Dirección de Información Agrícola y Forestal. (2011). Estimaciones Agrícolas MAGyP, por Departamento. Recuperado de http:// www.siia.gov.ar/index.php/series-por-tema/ agricultura.

Ferraro, R., Zulaica, L. y Echechuri, H. (2013). Perspectivas de abordaje y caracterización del periurbano de Mar del Plata, Argentina. Revista Letras Verdes, (13), 19-40.

Gareis, M.C. (2017). Estimación de la huella ecológica de Mar del Plata (Partido de General Pueyrredón), y su relación con el 
hábitat urbano (tesis doctoral). Lujan, Argentina, Universidad Nacional de Lujan.

Gareis, M.C., y Ferraro, R.F. (2013). Estimación de la subhuella de absorción de $\mathrm{CO}_{2}$ del partido de General Pueyrredón (Argentina), del año 2010. DELOS Revista Desarrollo Local Sostenible, 6(17), 1-22. Disonible en: http://ojs.fch.unicen.edu.ar/index.php/ revistaestcig/article/view/122

Gareis, M.C., y Ferraro, R.F. (2014). Actividad turística y emisiones de $\mathrm{CO}_{2}$. El caso de Mar del Plata. Revista Estudios Ambientales, 2(1), 43-58. Disponible en: http:// ojs.fch.unicen.edu.ar/index.php/estudiosambientales/article/view/13

Gareis, M.C., y Ferraro, R.F. (2015). Estimación de la subhuella de ambiente construido del partido General Pueyrredon, Argentina. Revista Cuaderno Urbano, 19(19), 57-72. Disponible en: https://revistas.unne.edu.ar/ index.php/crn/article/view/588

Gareis, M.C., y Ferraro, R.F. (2018). Lectura de las áreas urbanas en clave de Economía Ecológica. Geografia em Questão, 11(1), 60-74.

Gareis, M.C., y Ferraro, R.F. (2019). La Huella Ecológica del partido de General Pueyrredon (Argentina) como indicador biofísico del metabolismo urbano. Revista Iberoamericana de Economía Ecológica, 29(1), 114-138.

Gareis, M.C., Gonzalez Insúa, M., y Ferraro, R.F. (2016). Incidencia de los recuperadores en las subhuellas de RSU y papel y cartón. El caso de Mar de Plata, Argentina. Estudios Socioterritoriales Revista de Geografía (19), 63-77.

Instituto Nacional de Estadísticas y Censos (INDEC). (2010). Censo Nacional de Población, Hogares y Viviendas (CNPHyV) del año 2010. Recuperado de www.censo2010. indec.gov.ar

Ministerio de Agricultura, Ganadería y Pesca de la Nación (MAGyP). (2013). Anuario 2011. Ganados y Carnes. Recuperado de http://minagri.siia.gob.ar/site/ganaderia/ anuario/pdf/00_COMPLETO_2011.pdf
Rees, W. E. (1996). Indicadores territoriales de sustentabilidad. Ecología Política (12), 27-41.

Rodríguez Mellado, J.F. (2008). Indicador para la Sostenibilidad de la Actividad Urbanística: Balance $\mathrm{CO}_{2}$ producido/ $\mathrm{CO}_{2}$ absorbido de la Aglomeración Urbana de Sevilla. En CONAMA 9. Congreso Nacional del Medio Ambiente. Cumbre del Desarrollo Sostenible (pp. 1-20). Madrid, España.

Wackernagel, M., y Rees, W. (1996). Our Ecological Footprint: Reducing Human Impact on the Earth. Gabriola Island, BC: New Society Publishers.

Zulaica, L., Ferraro R., \& Vazquez, P. (2011). Análisis temporal de los usos de suelo en el periurbano de Mar del Plata y el partido de General Pueyrredon (1986-2009). En Actas del Primer Congreso de Tecnologías de la Información Geográfica y Cuarta Reunión de Usuarios de Tecnologías de la Información Geográfica del NEA (pp. 479-492). Resistencia, Chaco, Argentina.

\section{Notas}

1 Este trabajo se encuadra en una tesis de posgrado del Doctorado de la Universidad Nacional de Lujan en la Orientación Ciencias Sociales y Humanas (Gareis, 2017).

2 Licenciada en Diagnóstico y Gestión Ambiental, Doctora en Ciencias Sociales y Humanas, Investigadora Asistente del Consejo Nacional de Investigaciones Científicas y Técnicas con radicación en el Instituto de Ambiente de Montaña y Regiones Áridas de la Universidad Nacional de Chilecito. Instituto de Ambiente de Montaña y Regiones Áridas (IAMRA), Universidad Nacional de Chilecito (UNdeC). Investigadora Asistente CONICET. Instituto del Hábitat y del Ambiente (IHAM), Facultad de Arquitectura, Urbanismo y Diseño (FAUD), Universidad Nacional de Mar del Plata (UNMdP). 